\title{
Expresión de p53 y Bcl-2 en carcinoma ductal infiltrante de mama localmente avanzado. Su relación con el subtipo intrínseco molecular como factor pronóstico.
}

\author{
Liliana Castillo ${ }^{1}$, Félix Bonilla ${ }^{2}$, Aldo Reigosa ${ }^{3}$ y Ángel Fernándes 3,4 \\ ${ }^{1}$ Instituto de Oncología Dr. Miguel Pérez Carreño. Valencia, Venezuela. \\ ${ }^{2}$ Hospital Universitario Puerta de Hierro Majadahonda. Madrid, España. \\ ${ }^{3}$ Centro de Investigaciones Médicas y Biotecnológicas de la Universidad de Carabobo. \\ Facultad de Ciencias de la Salud. Universidad de Carabobo. Valencia, Venezuela. \\ ${ }^{4}$ Departamento de Ciencias Fisiológicas. Escuela de Ciencias Biomédicas \\ y Tecnológicas. Facultad de Ciencias de la Salud. Universidad de Carabobo. Valencia, \\ Venezuela.
}

Palabras clave: cáncer de mama; subtipo molecular; p53; Bel-2.

Resumen. En el cáncer de mama existen factores pronósticos y predictivos que pueden afectar la evolución de las mujeres con esta enfermedad. Por tal motivo, el presente estudio tiene como objetivo determinar la expresión inmunohistoquímica (IHQ), de las proteínas p53 y Bcl-2 en el tumor primario de 285 mujeres, con diagnóstico de carcinoma ductal infiltrante de mama, en seguimiento en el Instituto de Oncología Dr. Miguel Pérez Carreño de Valencia, Venezuela. La inmunoexpresión de ambas proteínas se relacionó con los subtipos intrínsecos moleculares del cáncer de mama definidos por IHQ: Luminal A, Luminal B, HER2 y Triple Negativo (TN), la supervivencia libre de enfermedad (SLE) y la supervivencia global (SG). Los tumores p53 negativo y Bel-2 positivo mostraron una mayor SLE y SG. En los tumores p53 negativo del subtipo Luminal A hubo una mayor SLE ( $\mathrm{p}=0,005)$, aunque en los tumores Luminales (A y $\mathrm{B})$, la SG fue mayor $(\mathrm{p}<0,05)$. Además, se presentó una mayor SLE en los casos Luminales y TN con expresión positiva de Bel-2, pero sin significancia estadística. Por el contrario, hubo un efecto pronóstico significativo de la expresión de Bel-2 en el subtipo TN, con SG media menor en comparación con la SG obtenida en los tumores con Bel-2 positivo. Finalmente, en función de los hallazgoos encontrados se concluye que la ausencia de expresión p53 y la expresión positiva de Bel-2 son factores de buen pronóstico, con significancia estadística en los tumores Luminales y TN, respectivamente.

Autor de correspondencia: Ángel Fernández. Departamento de Ciencias Fisiológicas. Escuela de Ciencias Biomédicas y Tecnológicas. Facultad de Ciencias de la Salud. Universidad de Carabobo. Valencia, Venezuela. Teléfono: +580241-8666243. Correo electrónico: aafernandez@uc.edu.ve. 


\title{
Expression of p53 and Bcl-2 in locally advanced infiltrating ductal carcinoma of the breast. Its relationship with the intrinsic molecular subtype as a prognostic factor.
}

\author{
Invest Clin 2018; 59 (4): 325 - 338
}

Key words: breast cancer; molecular subtype; p53; Bcl-2.

\begin{abstract}
In breast cancer there are prognostic and predictive factors that can affect the evolution of women with breast eancer. For this reason, the present study aims to determine the immunohistochemical expression (IHC) of p53 and Bel-2 proteins in the primary tumor of 285 women with a diagnosis of infiltrating ductal carcinoma of the breast under follow-up at the Dr. Miguel Oncology Institute. Pérez Carreño from Valencia, Venezuela. The immunoexpression of both proteins was related to the molecular intrinsic subtypes of breast cancer defined by IHC: Luminal A, Luminal B, HER2 and Triple Negative (TN), disease free survival (SLE) and overall survival (OS). The tumors p53 negative and Bel-2 positive showed a higher SLE and SG. In the p53 negative tumors of the Luminal A subtype there was a higher SLE $(\mathrm{p}=0.005)$, although in the Luminal tumors (A and $\mathrm{B}$ ) the OS was higher with $\mathrm{p}<0.05$. In addition, greater SLE was presented in Luminal and TN cases with positive Bcl-2 expression, but without statistical significance. On the contrary, there was a significant prognostic effect of the expression of Bel-2 in the TN subtype, with a lower mean SG compared to the SG obtained in tumors with positive Bel-2. Finally, based on the findings found, the absence of p53 expression and the positive expression of Bel-2 are good prognostic factors, with statistical significance in Luminal and TN tumors, respectively.
\end{abstract}

Recibido 17-05-2018 Aceptado 28-11-2018

\section{INTRODUCGIÓN}

El cáncer de mama constituye un problema de salud pública a nivel mundial y representa el tipo de cáncer más frecuente en el sexo femenino. En el año 2015 fueron diagnosticadas 1,7 millones de mujeres; medio millón de ellas murieron a causa de esta enfermedad, siendo más de la mitad de los casos diagnosticados en los países desarrollados: 370.000 casos al año en Europa $(27,4 \%)$ y 230.000 en Norteamérica $(31,3 \%)(1,2)$.

La Organización Panamericana de la Salud, en su publicación más reciente, indica que para el año 2012, 408.000 mujeres fueron diagnosticadas con cáncer de mama en Latinoamérica, de las cuales 92.000 fallecieron a causa de esta enfermedad (3). En Venezuela, el Anuario de Mortalidad del Ministerio del Poder Popular para la Salud, demostró que el carcinoma mamario es la principal causa de muerte por cáncer en mujeres, con un registro de 2060 casos durante el año 2013 y para el 2017 las proyecciones de la Sociedad Anticancerosa de Venezuela, estimaron 26.500 muertes como consecuencia del cáncer, con un aproximado de 53.000 casos nuevos (4).

La heterogeneidad del carcinoma de mama es un hecho bien reconocido y aceptado, que puede explicarse por su origen de células madre cancerosas con potencia- 
les evolutivos diferentes y un significativo número de alteraciones genéticas posibles, esto gracias a los estudios a nivel genómico, transcriptómico y proteómico $(5,6)$. Numerosos trabajos han confirmado la utilidad de los subtipos moleculares por su capacidad de predecir la evolución y la respuesta a diferentes tratamientos en el cáncer de mama, lo que ha contribuido a la medicina personalizada. Sin embargo, estos subtipos no son grupos homogéneos, ya que existen grupos híbridos o subtipos con importancia pronóstica y terapéutica que pueden definirse mediante la inmunohistoquímica (IHQ). Actualmente, desde el punto de vista IHQ, se reconocen los subtipos intrínsecos moleculares Luminal A, Luminal B, HER2 y Triple Negativo (TN) (7-9).

Utilizando la IHQ se han definidos factores pronósticos y predictivos, que pueden afectar la evolución del cáncer de mama, entre los que se incluyen las proteínas p53 y Bel-2 (10,11). La p53 es un factor de transcripción nuclear que se une al ácido desoxirribonucleico $(\mathrm{ADN})$, para regular la transcripción de determinados genes, jugando un papel en el control del ciclo celular a través de la detección de la célula en la fase G1, G2 o M, la iniciación de la reparación del $\mathrm{ADN}$ o la activación de la muerte celular por apoptosis. Esta respuesta se pierde ante el gen inactivado por mutación, el cual produce una proteína defectuosa que ejerce un efecto contrario, provocando la multiplicación de células genómicante alteradas y el crecimiento tumoral, por lo que se considera un factor de mal pronóstico en los diferentes subtipos moleculares (12-14).

Por su parte, la proteína Bel-2 tiene una función anti-apoptótica e inhibe la muerte celular, lo que resulta en la supervivencia celular prolongada. Se expresa con frecuencia en el epitelio mamario normal y está regulada positivamente por el estrógeno $(15,16)$. Asimismo, ha demostrado ser un predictor independiente de pronóstico favorable, aunque con resultados inconsistentes en diferentes estudios, en relación con los subtipos in- trínsecos moleculares y por ello sigue siendo controversial sobre todo en los tumores TN (receptores hormonales y HER2 negativos), donde ha mostrado resultados contradictorios en diferentes publicaciones $(17,18)$.

Por tales razones, esta investigación tiene como objetivo general, determinar a través de la IHQ, el perfil de inmunoexpresión de las proteínas p53 y Bcl-2 en el tumor primario, relacionando su expresión con los subtipos intrínsecos moleculares del carcinoma de mama, la supervivencia libre de enfermedad (SLE) y la supervivencia global (SG) de las pacientes evaluadas, considerando que una mejor caracterización inmunofenotípica de los tumores, permitirá avanzar en el establecimiento de diferentes categorías con distinto pronóstico y con ello, un abordaje terapéutico más efectivo.

\section{PACIENTES Y MÉTODOS}

El presente estudio se realizó en pacientes diagnosticadas con carcinoma ductal infiltrante de mama, en seguimiento en el Instituto de Oncología Dr. Miguel Pérez Carreño (IOMPC) de Valencia, Venezuela. Se determinó la expresión inmunohistoquímica (IHQ) de p53 y Bcl-2 y se compararon los resultados obtenidos con la supervivencia libre de enfermedad (SLE), la supervivencia global (SG) y los subtipos intrínsecos definidos por IHQ, de acuerdo a la expresión del receptor de estrógeno (RE), del receptor de progesterona (RP) y del receptor del factor de crecimiento epidérmico 2 (HER2).

La SLE en meses, se definió como el tiempo transcurrido desde el tratamiento radical (cirugía o radioterapia), hasta la fecha de la recaída bien sea local o a distancia (en caso de haber ocurrido). Por su parte, la SG en meses se consideró como el tiempo transcurrido desde el diaǵnóstico, hasta la fecha de la muerte, atribuida o no al cáncer de mama. Se realizó el seguimiento clínico hasta 165 meses y el tiempo de supervivencia, en caso de haber ocurrido la muerte antes de los 60 meses. 
Sujetos del estudio. Las pacientes en esta investigación fueron diagnosticadas con cáncer de mama en el IOMPC entre los años 2005 y 2012, con un seguimiento hasta 2017. Con la aprobación del Comité de Ética y de la Comisión de Investigación del IOMPC, se conformó una serie no aleatoria, de tipo intencional, con 285 mujeres, de acuerdo a los siguientes criterios de inclusión: a) diaǵnóstico de carcinoma ductal infiltrante de mama sin otra especificación, o mixto, con componente ductal predominante, $b$ ) resultados previos de los niveles de expresión IHQ del RE, RP y HER2 y c) disponibilidad de las preparaciones histológicas y bloques de parafina respectivos correspondientes al diaǵnóstico inicial.

Inmunohistoquímica. Las muestras tisulares se fijaron en formol y se incluyeron en parafina siguiendo los métodos convencionales en el Servicio de Anatomía Patológica del IOMPC. De los bloques de parafina se obtuvieron secciones histológicas de $4 \mu \mathrm{m}$ de espesor, que fueron recogidas en láminas portaobjetos tratadas previamente con polyL-lisina, para evitar el desprendimiento de los cortes. Posteriormente, los cortes fueron trasladados al Centro de Investigaciones Médicas y Biotecnológicas de la Universidad de Carabobo (CIMBUC), en donde se procedió a realizar la técnica de la IHQ.

Luego de la desparafinación e hidratación de las muestras, la recuperación antigénica se realizó en el Isotemp Water Bath (modelo Isotemp 205, Fisher Scientific ${ }^{\circledR}$. Estados Unidos) con un tampón Tris-EDTA (10 mM Tris-Base, $1 \mathrm{mM}$ EDTA, 0,05\% Tween 20, pH 9,0, Dako. Estados Unidos) a $95^{\circ} \mathrm{C}$. Seguidamente, los cortes se lavaron en tampón Tris-HCl $(50 \mathrm{mM}$ Tris-HCl, $150 \mathrm{mM} \mathrm{NaCl}$, 0,05\% Tween 20, pH 7,6, Dako. Estados Unidos) a temperatura ambiente. El bloqueo de la peroxidasa endógena se realizó con peróxido de hidrógeno al 3\% en metanol. Consecutivamente, se realizó la incubación con el anticuerpo primario (p53, clon D07, dilución 1:100 y Bel-2, clon 124, dilución 1:40, ambos marca Dako, Estados Unidos), de acuerdo al protocolo indicado por la casa comercial. Las muestras se lavaron en tampón Tris-HCl y se añadió el anticuerpo secundario estreptavidina-biotina-peroxidasa (Envision, Dako, Estados Unidos). Finalmente, la inmunotinción se reveló mediante la aplicación del cromógeno diaminobencidina $(0,05 \%$ tetrahidrocloruro de diaminobencidina, $50 \mathrm{mM}$ Tris-HCl, pH 7,4). El montaje de los cortes se efectuó con resina sintética (DPX), para su visualización posterior en el microscopio (marca Zeiss Axiostar Plus, Estados Unidos). En la valoración de la expresión de ambas proteínas, se consideró un resultado positivo cuando existía $>10 \%$ de inmunoexpresión y se refirió como el resultado negativo, cuando había expresión en el $10 \%$ o menos de las células tumorales.

Análisis estadístico. El análisis de los datos recogidos se realizó mediante el paquete estadístico SPSS (Statistical Package for Social Sciences, versión 22). Los resultados se expresaron como media y desviación estándar en el caso de variables cuantitativas y como número absoluto y porcentaje en las cualitativas. La asociación entre las variables clínico-patológicas, subtipos intrínsecos moleculares y marcador inmunohistoquímico se analizó con la prueba de Chi cuadrado y el test exacto de Fisher. El estudio de supervivencia se realizó mediante el método de Kaplan-Meier. Se consideraron significativos los valores de $\mathrm{p}<0,05$.

\section{RESULTADOS}

La edad media de las pacientes en el momento del diagnóstico fue de 51,6 años. El estadio clínico más frecuente fue el III e histológicamente los tumores en su mayoría fueron moderadamente diferenciados. El seguimiento de las pacientes de la serie osciló entre 5 y 165 meses. La media para la SLE se ubicó en 70,4 \pm 41,4 meses y la SG media fue mayor con $87,8 \pm 38,2$ meses. Más del 30\% de las pacientes fallecieron durante el tiempo de seguimiento. Los principales datos clínicopatológicos de las pacientes incluidas en este estudio se detallan en la Tabla I. 
TABLA I

CARACTERÍSTICAS CLÍNICO-PATOLÓGICAS DE LA SERIE

\begin{tabular}{|c|c|c|}
\hline \multicolumn{3}{|l|}{ Variable } \\
\hline Edad (años): media (rango) & -.- & $51,6(26-86)$ \\
\hline & & $\mathrm{n}(\%)$ \\
\hline Grupos etarios & $\leq 40$ & $53(18,6)$ \\
\hline \multirow{3}{*}{ (edad en años) } & $41-50$ & $94(33,0)$ \\
\hline & $51-60$ & $75(26,3)$ \\
\hline & $\geq 61$ & $63(22,1)$ \\
\hline \multirow[t]{4}{*}{ Estadio clínico } & I & $\ldots$ \\
\hline & II & $104(36,5)$ \\
\hline & III & $168(58,9)$ \\
\hline & IV & $13(4,6)$ \\
\hline \multirow[t]{3}{*}{ Grado histológico } & $\mathrm{I}$ & $53(18,6)$ \\
\hline & II & $161(56,5)$ \\
\hline & III & $71(24,9)$ \\
\hline \multirow[t]{4}{*}{ Subtipos intrínsecos moleculares } & Luminal A & $70(24,6)$ \\
\hline & Luminal B & $116(40,7)$ \\
\hline & HER2 & $21(7,4)$ \\
\hline & Triple Negativo & $78(27,3)$ \\
\hline \multirow[t]{2}{*}{ Estado } & Fallecida & $99(34,7)$ \\
\hline & Viva & $186(65,3)$ \\
\hline $\begin{array}{l}\text { Supervivencia libre de enfermedad } \\
\quad(\text { media en meses } \pm \text { DE) }\end{array}$ & -- & $70,4 \pm 41,4$ \\
\hline $\begin{array}{l}\text { Supervivencia global } \\
\text { (media en meses } \pm \text { DE) }\end{array}$ & --- & $87,8 \pm 38,2$ \\
\hline
\end{tabular}

La ausencia de expresión de la proteína p53 predominó en los subtipos Luminales y HER2, no obstante, en el subtipo TN más del $50 \%$ expresó la proteína p53 $(\mathrm{p}<0,05)$. Asimismo, el 68,6\% de los tumores Luminal A expresó la proteína Bcl-2, aunque el 53,4\% del subtipo Luminal B era Bel-2 negativo. En los tumores HER2 y TN, predominaron los casos Bcl-2 negativo ( $\mathrm{p}<0,05)$ (Tabla II).

Los tumores p53 negativo, mostraron un menor índice de recaída de la enfermedad, con una media de SLE de 94,4 \pm 4,2 meses comparados con 82,2 $\pm 5,3$ meses obtenidos en los casos p53 positivo $(\mathrm{p}<0,05)$. De igual forma, se obtuvo una mayor SG media de 122,6 $\pm 4,1$ meses en los casos p53 negativo versus la $\mathrm{SG}$ de 113,9 \pm 5,6 meses alcanzada en los tumores p53 positivo $(\mathrm{p}=0,035)$ (Fiǵ. 1). En relación al Bcl-2, la expresión positiva de Bcl-2 influyó en la SLE, al obtener una media de 100,8 \pm 5,5 meses a diferencia de los casos Bel-2 negativo, en donde la SLE fue de 79,8 \pm 5,6 meses $(\mathrm{p}<0,05)$. De igual forma, su expresión se asoció a una SG media de 140,2 $\pm 5,1$ a diferencia de la SG de 102,3 \pm 5,6 meses obtenida en los tumores Bcl-2 negativo ( $\mathrm{p}<0,05)$ (Fig. 2).

TABLA II

EXPRESIÓN DE LAS PROTEÍNAS P53 Y BCL-2 Y SU ASOCIACIÓN CON LOS SUBTIPOS INTRÍNSECOS MOLECULARES DEL CARCINOMA DE MAMA

\begin{tabular}{ccccccc}
\hline \multirow{2}{*}{ Marcador } & \multirow{2}{*}{ Expresión } & \multicolumn{4}{c}{$\mathrm{n}(\%)$} & \multirow{2}{*}{ p $^{*}$} \\
\cline { 3 - 6 } & & Luminal A & Luminal B & HER2 & TN & \\
\hline \multirow{2}{*}{ p53 } & Neǵativo & $51(72,9)$ & $69(59,5)$ & $18(85,7)$ & $38(48,7)$ & \multirow{2}{*}{0,004} \\
& Positivo & $19(27,1)$ & $47(40,5)$ & $3(14,3)$ & $40(51,3)$ & \\
Bel-2 & Neǵativo & $22(31,4)$ & $62(53,4)$ & $14(66,7)$ & $57(73,1)$ & $<0,001$ \\
& Positivo & $48(68,6)$ & $54(46,6)$ & $7(33,3)$ & $21(26,9)$ & $<$ \\
\hline
\end{tabular}

$\mathrm{TN}=$ Triple Negativo. $\mathrm{p}^{*}=$ Prueba de $\mathrm{Chi}^{2}$. 

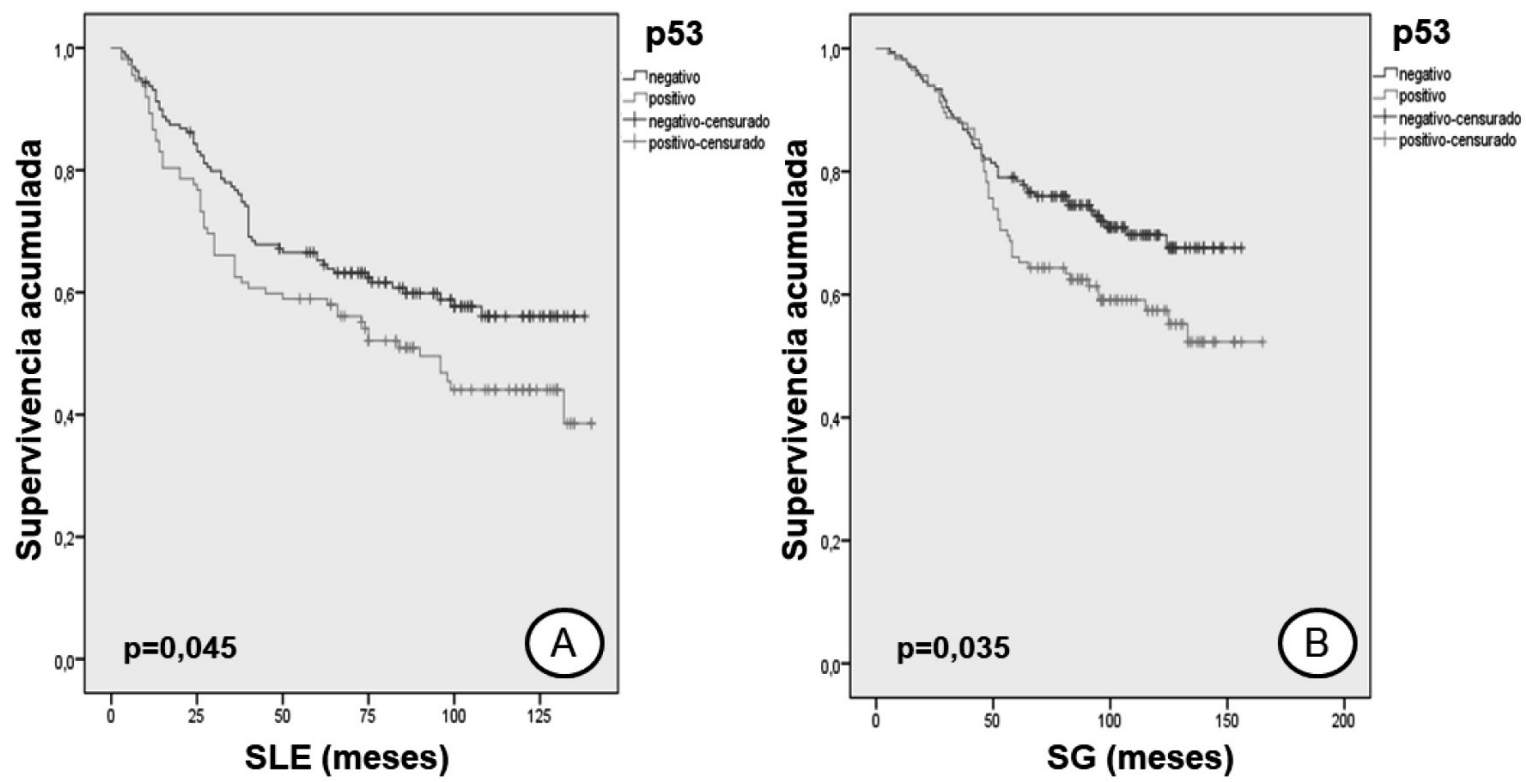

Fig. 1. Supervivencia libre de enfermedad (SLE, A) y supervivencia global (SG, B) media en la expresión de la proteína P53 determinada mediante inmunohistoquímica.
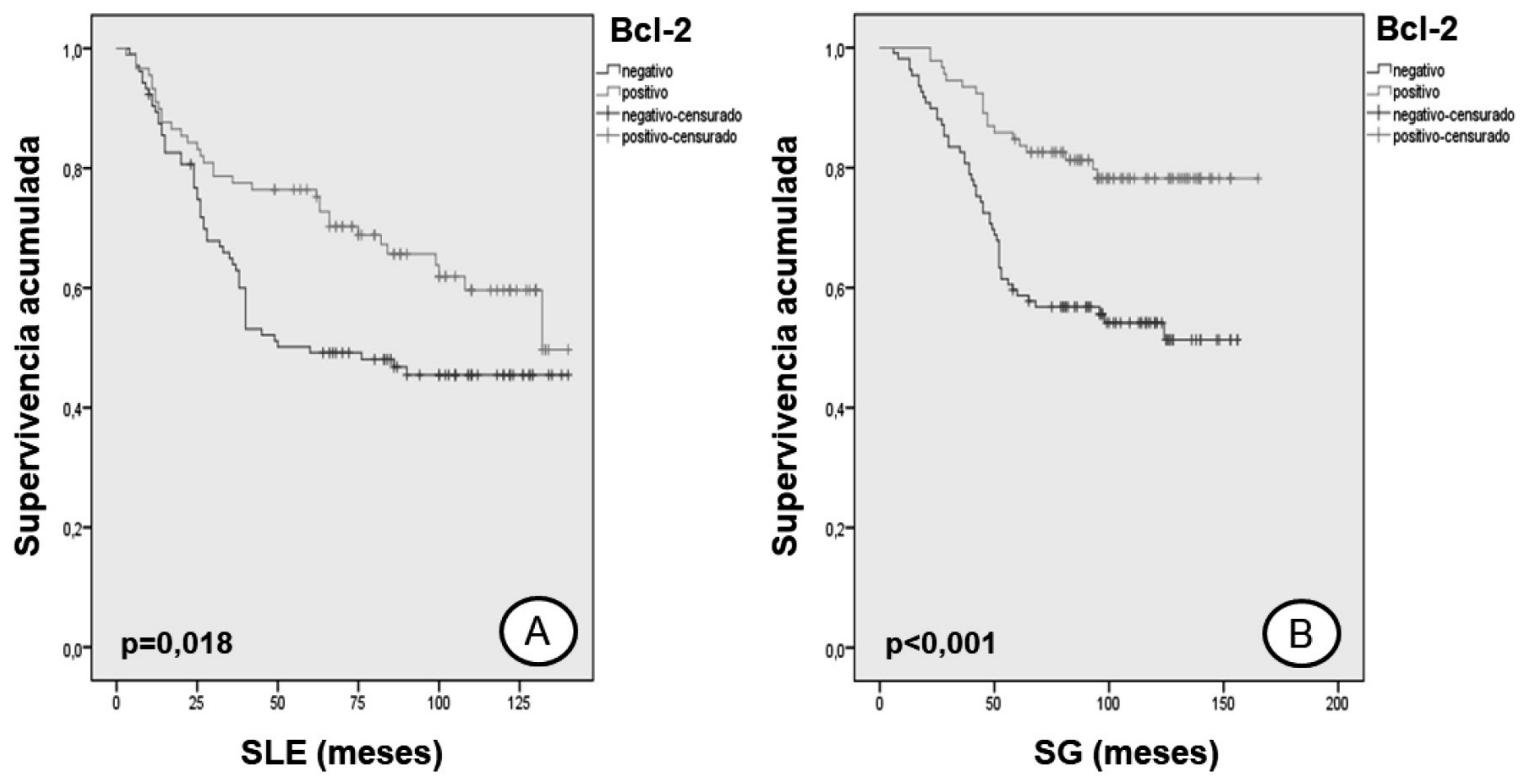

Fig. 2. Supervivencia libre de enfermedad (SLE, A) y supervivencia global (SG, B) media en la expresión de la proteína BCL-2 determinada mediante inmunohistoquímica. 
En el análisis de supervivencia de los subtipos intrínsecos de acuerdo a la expresión de p53 mediante IHQ, se obtuvo que en los tumores Luminal A hubo una mayor SLE de las pacientes con expresión negativa de p53, con una media de 107,7 $\pm 6,2$ meses contra 78,2 \pm 11,2 meses de los casos p53 positivo $(\mathrm{p}=0,005)$. Aunque se observó un comportamiento similar en los subtipos Luminal B y HER2, dichos hallazgos no alcanzaron la significancia estadística. Por el contrario, en los tumores $\mathrm{TN}$, la recidiva se presentó más tardía en los casos p53 positivo, con una media de $88,4 \pm 9,0$ meses en comparación a los 75,4 \pm 9,6 meses de los subtipos TN con p53 negativo, sin diferencia estadística significativa (Fig. 3).

Por otra parte, se evidenció una mayor SLE en las pacientes con tumores Luminales y TN, con expresión positiva de Bcl-2, aunque sin significancia estadística. Comportamiento contrario se obtuvo en los subtipos HER2, en donde la SLE disminuyó al hacerse negativo el p53 en las células tumorales, sin embargó, el bajo número de casos con estos tumores en la serie, limitó el siǵnificado del hallazgo (Fig. 4).
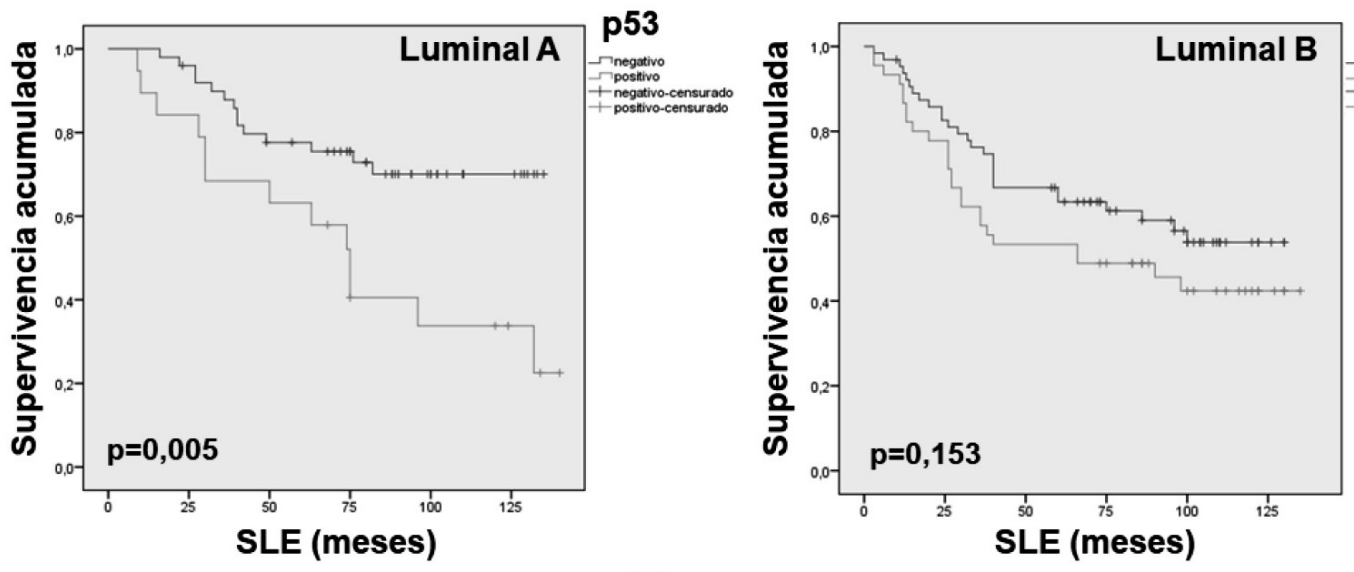

p53
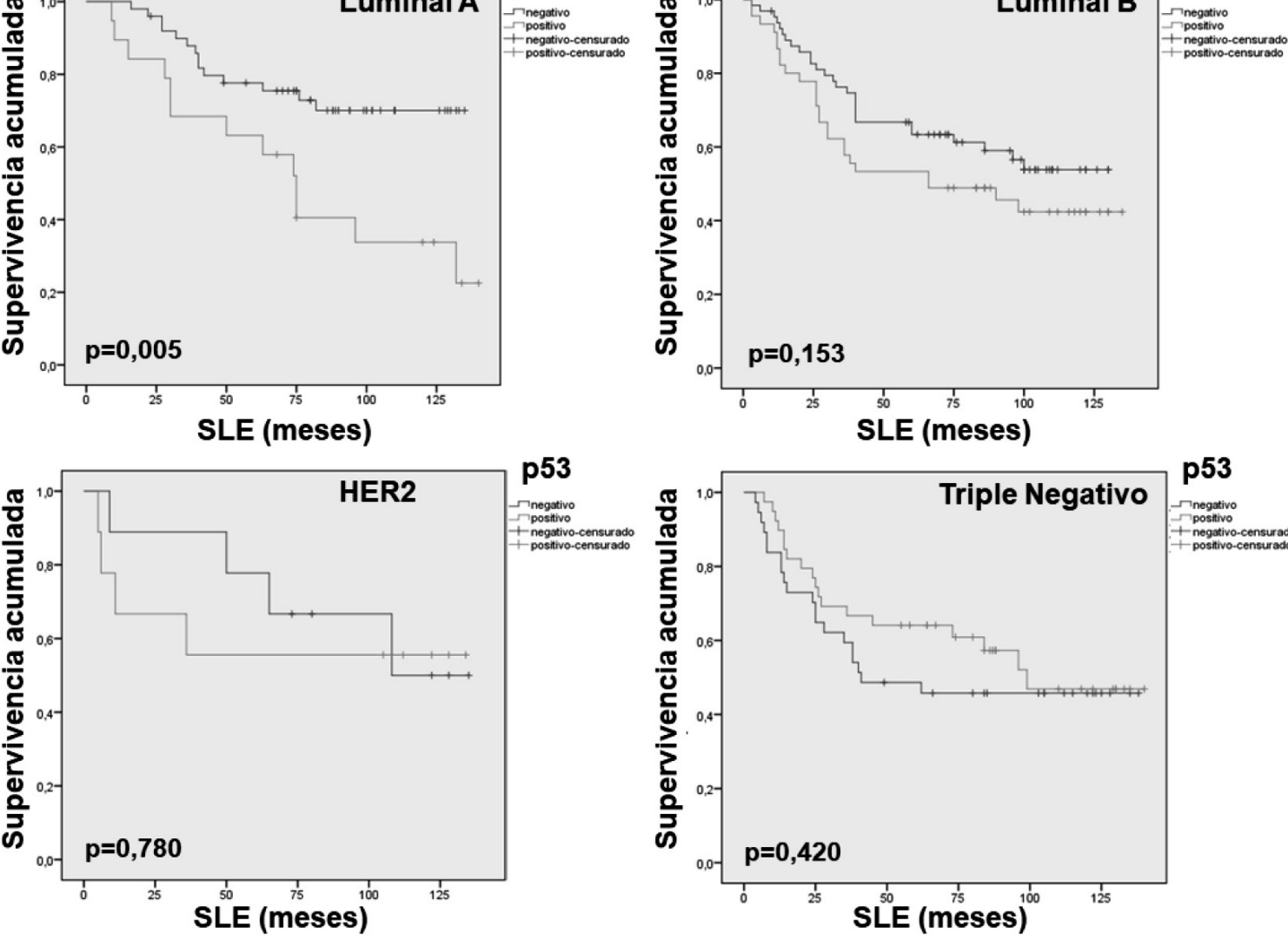

p53
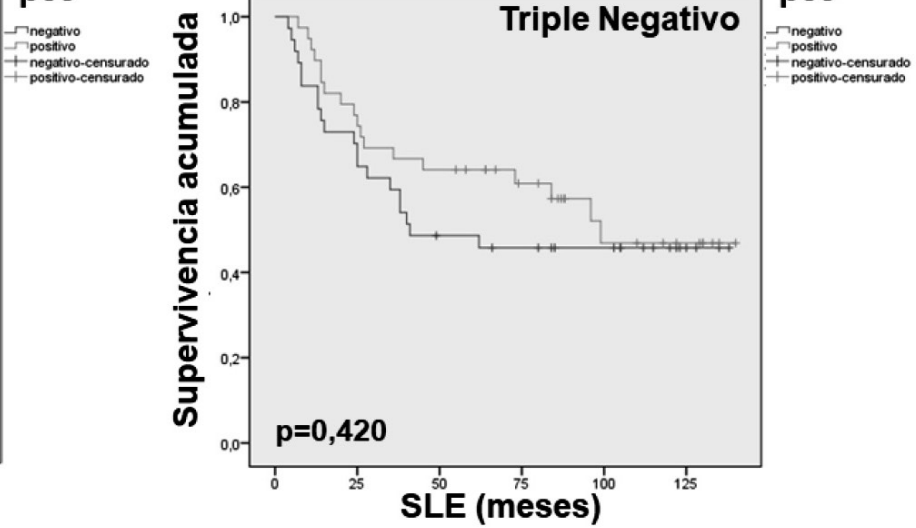

Fig. 3. Supervivencia MEDIA libre de enfermedad en los subtipos INTRÍNSECOS moleculares según la expresión de la proteína p53 determinada mediante inmunohistoquímica. 

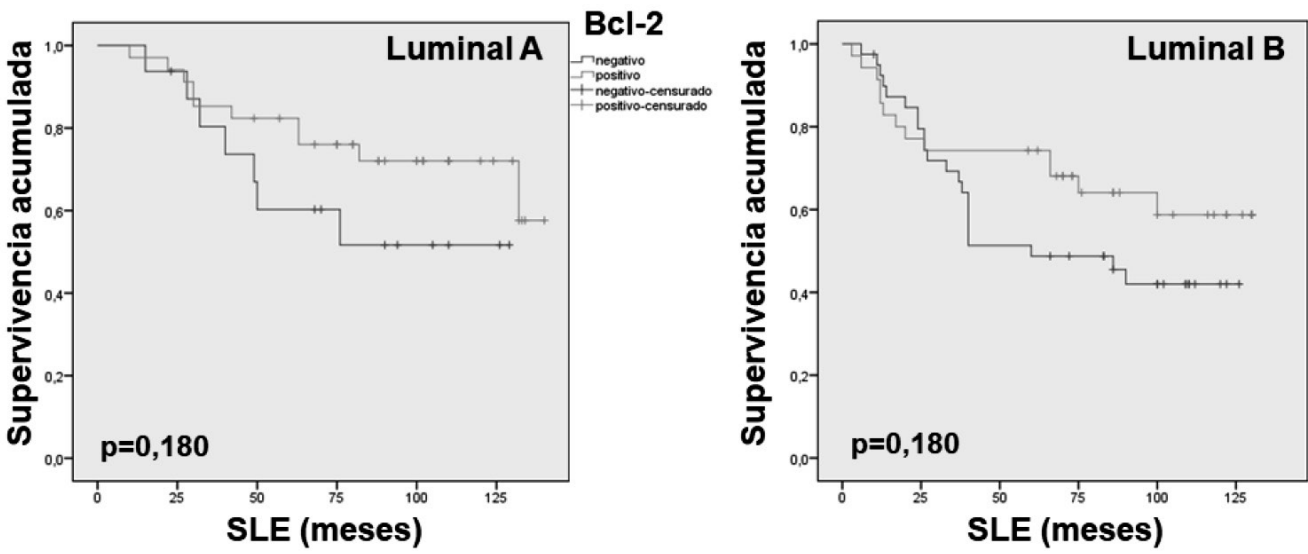

$\mathrm{Bcl}-2$
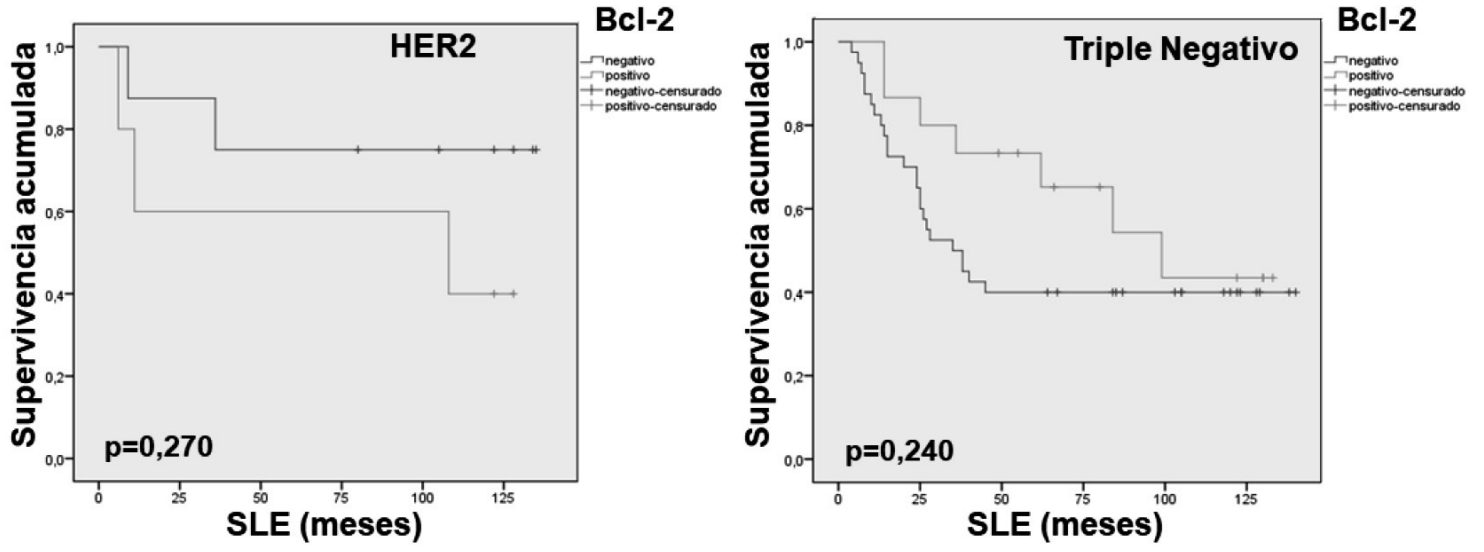

Fig. 4. Supervivencia media libre de enfermedad en los subtipos intrínsecos moleculares según la expresión de la proteína BCL-2 determinada mediante inmunohistoquímica.

Del mismo modo, se obtuvo una mayor SG en las pacientes con ausencia de expresión de p53 en relación a las mujeres cuyos tumores expresaron p53 y pertenecían a los subtipos intrínsecos moleculares Luminal A y Luminal B, con valor de $\mathrm{p} \leq 0,01$, respectivamente. No se encontró significancia estadística entre la SG y los subtipos HER2 y TN (Fig. 5).

Asimismo, hubo un efecto pronóstico significativo de la expresión de Bel-2 en el subtipo TN, con $\mathrm{SG}$ media significativamen- te menor ( $83 \pm 9,2$ meses), en comparación con la SG (131,4 \pm 6,2 meses) obtenida en los tumores TN con Bel-2 positivo, con $\mathrm{p}<0,05$. Esta tendencia se observó en los otros subtipos intrínsecos, pero sin significancia estadística (Fig. 6).

Finalmente, las Figuras 7 y 8 muestran ejemplos representativos de los patrones de expresión inmunohistoquimica observados en los marcadores analizados en este estudio. 

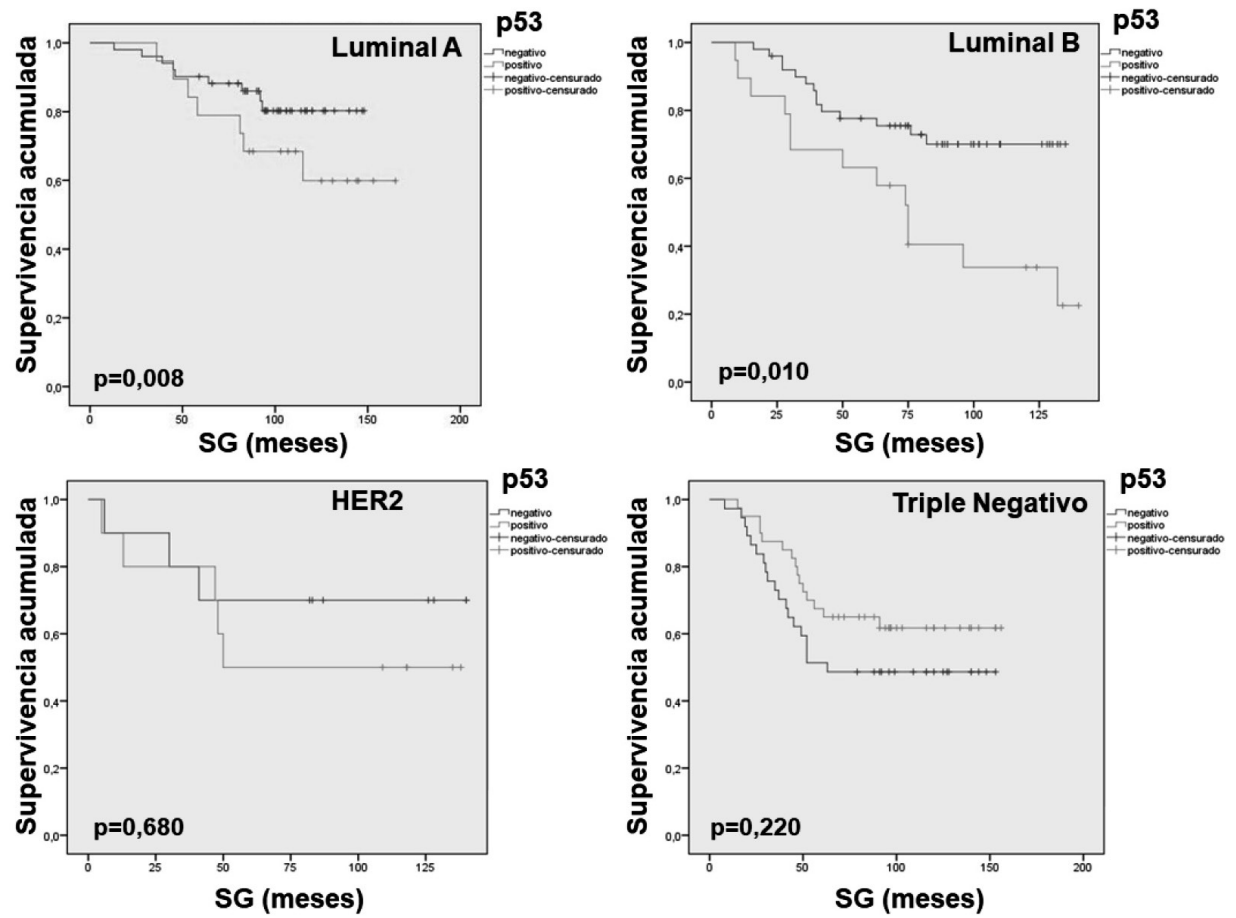

Fig. 5. Supervivencia GLOBAL media en los subtipos INTRÍNSECOS moleculares según la expresión de la proteína p53 determinada mediante inmunohistoquímica.
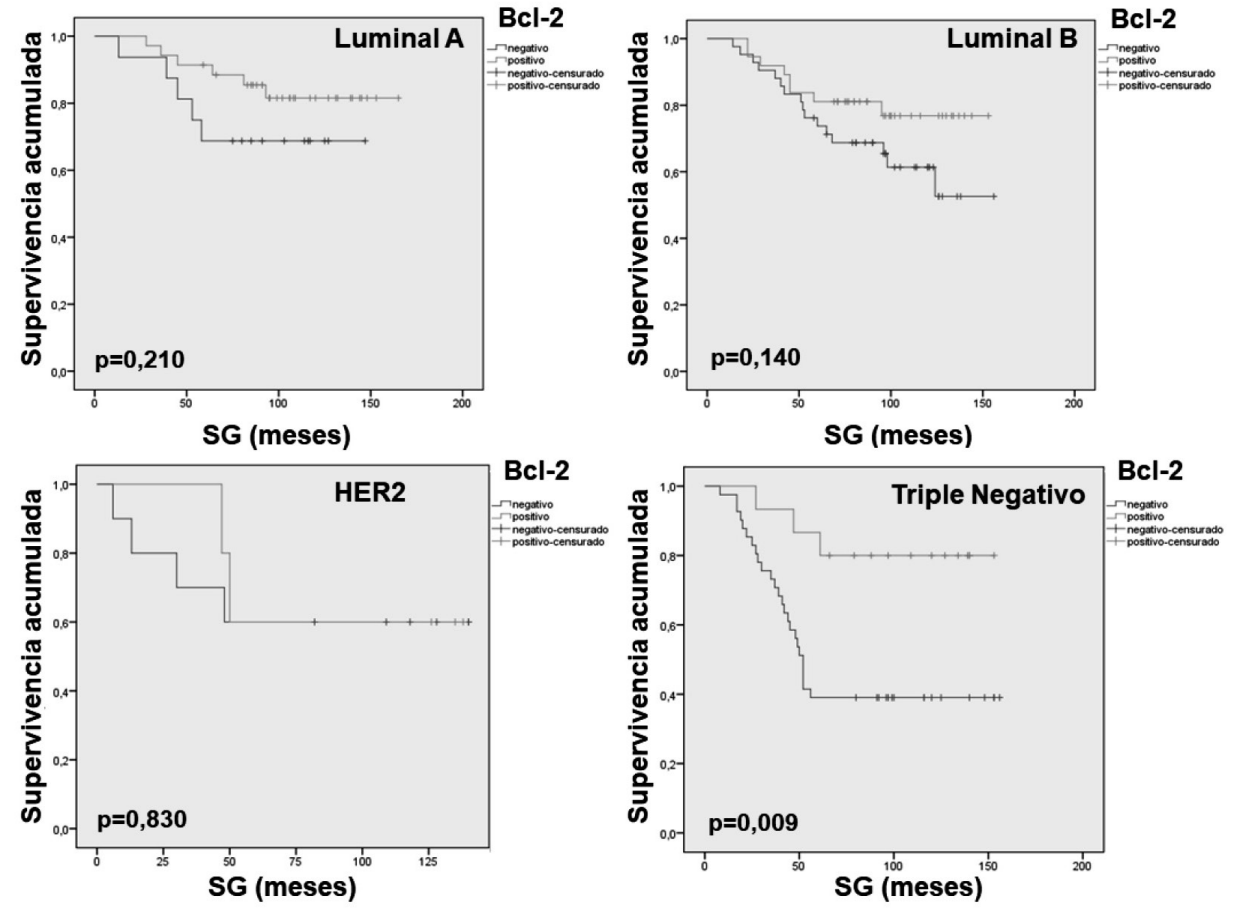

Fig. 6. Supervivencia GLOBAL media en los subtipos INTRÍNSECOS moleculares intrínsecos según la expresión de la proteína BCL-2 determinada mediante inmunohistoquímica. 


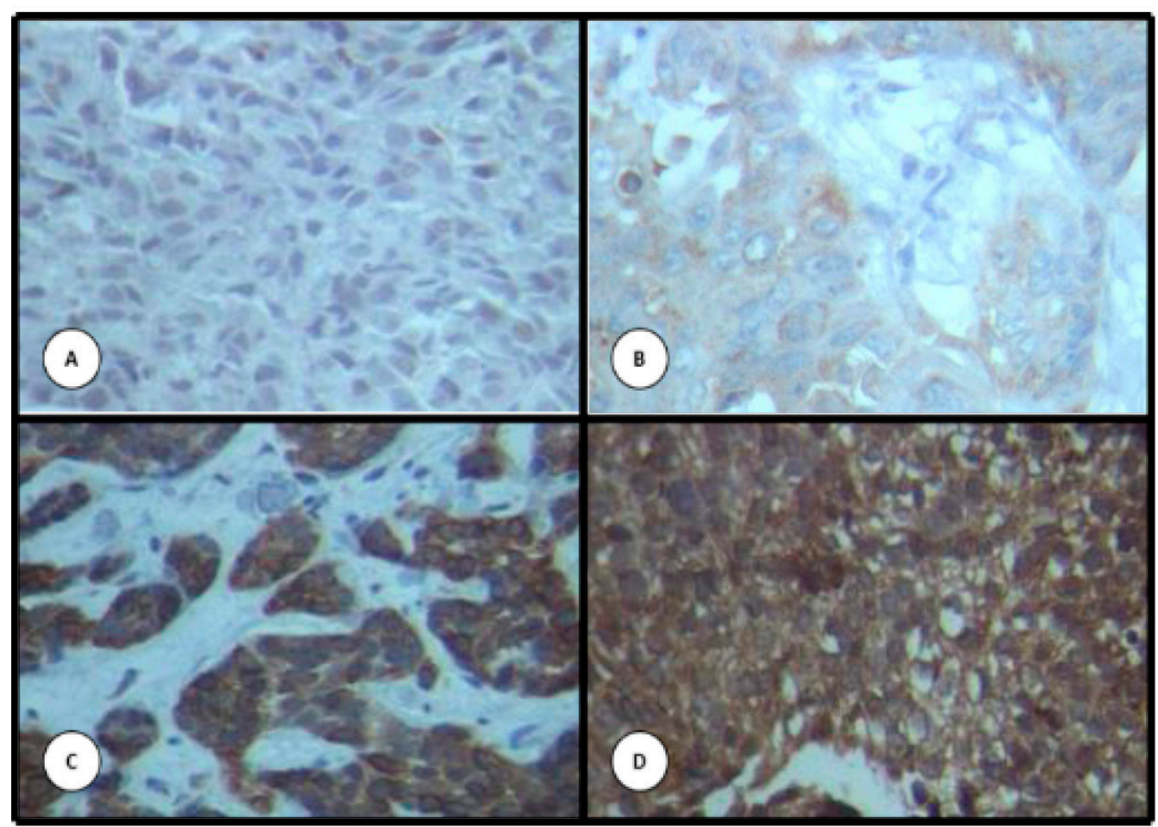

Fig. 7. Imágenes de inmuno-expresión de Bel-2. A) Reacción negativa. B) Expresión en pocas células aisladas. C) Marcaje en la casi totalidad de las células. D) Expresión en el 100\% de las células neoplásicas. Aumento original de 200X.

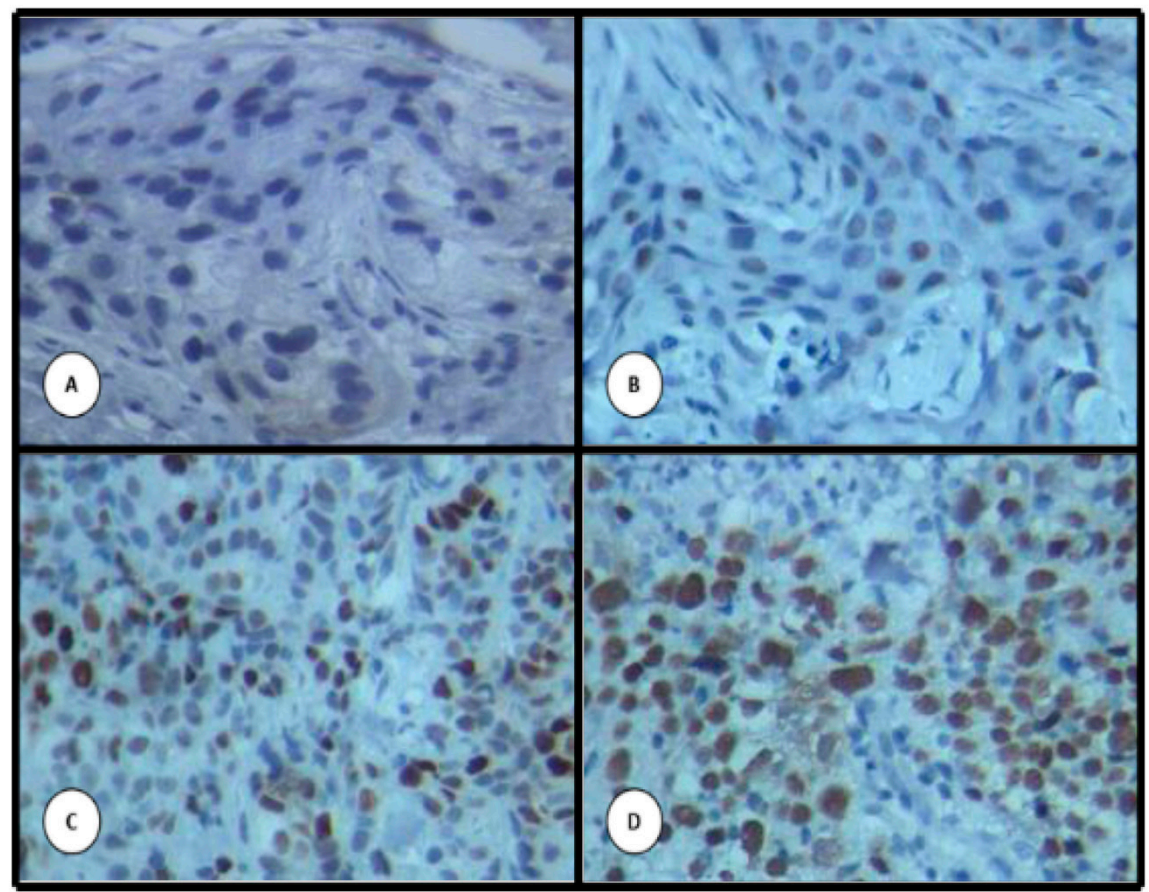

Fig. 8. Imágenes de inmuno-expresión de p53. A) Reacción negativa. B) Expresión en el 20\% de las células. C) Marcaje en el 60\% de las células. D) Expresión en el 95\% de las células neoplásicas. Aumento original de $200 \mathrm{X}$. 


\section{DISCUSIÓN}

Las características clínico-patológicas de la serie de pacientes con diagnóstico de cáncer de mama estudiada, tales como la edad, grado histológico y estadio clínico, fueron comparables con lo encontrado en el trabajo de Reigosa y col. (22). En relación a la clasificación molecular del carcinoma de mama, llama la atención que el Luminal $\mathrm{B}$, resultó ser el más frecuente, lo cual difiere con otros trabajos que han reportado que el Luminal A es el que predomina sobre los otros subtipos $(11,22,23)$. En la serie, la mayor frecuencia de casos correspondió a tumores HER2, comparable a lo publicado en los otros estudios $(11,23)$. En cuanto a los casos TN, la frecuencia fue bastante mayor que en mujeres caucásicas, en quienes la media es de $16 \%$, pero comparable con las cifras referidas a mujeres afroamericanas e hispánicas $(11,22,23)$. En este estudio no se indagó expresamente la raza de las pacientes, sin embargo, las mujeres que acuden al IOMPC, son en su gran mayoría de bajos recursos económicos y de raza predominantemente mestiza o hispana.

Los subtipos intrínsecos moleculares mostraron curvas de supervivencia similares a las descritas en la gran mayoría de las publicaciones $(10,22)$. Cabe destacar, que el Luminal A presentó el mejor pronóstico con respecto a los otros subtipos, pero sin significancia estadística. El Luminal B mostró una supervivencia intermedia y los subtipos HER2 y TN, fueron los de peor pronóstico.

Se encontró una asociación estadísticamente significativa entre la inmunoexpresión de p53 y los subtipos intrínsecos moleculares, específicamente se evidenció que en los tumores Luminal A, predominaron los casos p53 negativo, lo cual se relacionó con una mayor SLE y SG y por ende con un buen pronóstico; por el contrario, en el TN predominaron los tumores p53 positivo con SLE y SG menor, asociándose a un mal pronóstico, hallazǵos que coinciden con los resultados de otros trabajos publicados $(13,16,17,23)$.
En este trabajo, la frecuencia de la expresión IHQ de Bel-2 difirió entre los subtipos intrínsecos moleculares del carcinoma de mama. Estos hallazgos contrastan con los encontrados por Seong y col. (24), quiénes informaron que en 1304 pacientes con cáncer de mama la expresión de Bcl-2 fue del $54,4 \%$. Por subtipos, reportaron que el Bcl-2 se expresó en el $74 \%$ de los pacientes con Luminal A, 11\% Luminal B, 3\% HER2 y 13\% en pacientes con cáncer de mama TN.

Por otra parte, también se evidenció que la expresión de Bcl-2 tiene un efecto pronóstico según los subtipos intrínsecos redefinidos en el Consenso Internacional de Expertos, St. Gallen 2015 (25). La expresión de Bcl-2 fue un marcador de buen pronóstico en función a la SG en todos los subtipos intrínsecos moleculares, con significancia estadística sólo en el subtipo TN, aunque con SG media significativamente menor en comparación de la SG obtenida en los tumores Luminal A con Bel-2 positivo, lo que parece reflejar el efecto indirecto de los receptores de hormonas expresados en este último subtipo de cáncer de mama $(26,27)$. Estos hallazǵos están en consonancia con otros estudios (24-26), los cuales informaron que los tumores Luminal A con alta expresión de Bel-2 tienen mejor evolución en comparación a los otros subtipos de cáncer de mama.

Aunque no hubo una relación signnificativa, en nuestro estudio las pacientes con tumores Luminal B y sobreexpresión de Bcl-2 tuvieron un mejor pronóstico. A pesar que este subtipo expresa un alto nivel de Ki-67, la expresión de Bcl-2 parece jugar un papel anti-proliferativo que conlleva un resultado clínico favorable en comparación con el cáncer de mama con expresión Bcl-2 negativa. Por otra parte, una baja frecuencia de expresión de Bel-2, se detectó en los tumores HER2, lo que fue consistente con los hallazgos de otros estudios $(24,26,27)$.

Nuestro resultado muestra que en el subtipo TN, la ausencia de expresión de Bcl2 tiende a asociarse a un peor efecto pronóstico. Este resultado no concuerda con otros 
estudios que informaron que la baja expresión de Bcl-2, está asociada con buenos resultados en TN y que sugirieren que una alta tasa de expresión de Bel-2, es un importante predictor independiente de peor pronóstico en pacientes con cáncer de mama TN (2427). En consonancia con los hallazgos de este trabajo, en el estudio de Dawson y col. (28) concluyeron que la expresión de Bel-2, está relacionada con una mejor supervivencia ǵlobal en todos los subtipos de cáncer de mama, incluido el TN. De igual forma, Abdel-Fatah y col. (29), mostraron que la baja expresión de Bcl-2 resultó en una evolución pobre en pacientes con TN.

En resumen y aunque el papel de Bel-2 en TN permanece controvertido, la mayoría de los estudios han concluido que la ausencia de expresión en pacientes con receptores hormonales negativos o triple negativos se asocia con un mal pronóstico, lo que sugiere que la naturaleza anti-apoptótica de Bcl-2 se exhibe claramente en tales condiciones (29-31).

Finalmente, en función de los hallazgoos encontrados, se concluye que la ausencia de expresión p53 y la expresión positiva de Bel2 , son factores de buen pronóstico, con significancia estadística en los tumores Luminales y TN, respectivamente. Se espera que investigaciones adicionales en la población de mujeres venezolanas, puedan respaldar aún más los resultados de este estudio, al verificar el mecanismo de acción por el cual la expresión de p53 y Bel-2 influye en el pronóstico de los subtipos intrínsecos moleculares del carcinoma de mama.

\section{AGRADECIMIENTOS}

A todo el personal técnico y médico que labora en el Servicio de Oncología Médica, Anatomía Patológica e Historias Médicas del Instituto de Oncología Dr. Miguel Pérez Carreño y del Centro de Investiǵaciones Médicas y Biotecnológicas de la Universidad de Carabobo.

\section{REFERENCIAS}

1. Organización Mundial de la Salud. Cáncer. Temas de Salud. Mayo de 2016. [Documento en línea, consultado en junio de 2017]. Disponible en: http://www.who.int/ cancer/prevention/diagnosis-sereening/ breast-cancer/en/.

2. Jemal A, Bray F, Center MM, Ferlay J, Ward E, Forman D. Global eancer statistics. CA Cancer J Clin 2011; 61:69-90.

3. Organización Panamericana de la Salud. Cáncer de mama. Octubre de 2016 [Documento en línea, consultado en mayo 2017]. Disponible en: http://www.paho.org/hq/ index.php?option $=$ com_content\&view $=$ ar ticle\&id $=5041 \% 3 \mathrm{~A} 2011$-breast-cancer\&ca tid $=1872 \% 3$ Acancer \&Itemid $=3639 \&$ lang $=$ es.

4. Ministerio del Poder Popular para la Salud. Anuario de mortalidad 2013. Venezuela. Diciembre 2015. [Documento en línea, consultado en Julio 2017]. Disponible en: https://www.ovsalud.org/descargas/publicaciones/documentos-oficiales/AnuarioMortalidad-2013.pdf.

5. Melchor L, Saucedo-Cuevas LP, MuñozRepeto I, Rodríguez-Pinilla SM, Honrado E, Campoverde A, Palacios J, Nathanson KL, García MJ, Benítez J. Comprehensive characterization of the DNA amplification at $13 \mathrm{q} 34$ in human breast eancer reveals TFDP1 and CUL4A as likely candidate target genes. Breast Cancer Res 2009; 11:R86.

6. Park SY, Lee HE, Li H, Shipitsin M, Gelman R, Polyak K. Heterogeneity for stem cell-related markers according to tumor subtype and histologic stage in breast cancer. Clin Cancer Res 2010; 16:876-887.

7. Anders CK, Carey LA. Biology, metastatic patterns, and treatment of patients with triple-negative breast cancer. Clin Breast Cancer 2009; 9:73-81.

8. Sircoulomb F, Bekhouche I, Finetti P, Adélaïde J, Ben Hamida A, Bonansea J, Raynaud S, Innocenti C, Charafe-Jauffret E, Tarpin C, Ben Ayed F, Viens P, Jacquemier J, Bertucei F, Birnbaum D, Chaffanet M. Genome profiling of ERBB2-amplified breast eancers. BMC Cancer 2010; 10:539557. 
9. Perou CM, Sørlie T, Eisen MB, van de Rijn M, Jeffrey SS, Rees CA, Pollack JR, Ross DT, Johnsen H, Akslen LA, Fluge O, Pergamenschikov A, Williams C, Zhu SX, Lønning PE, Børresen-Dale AL, Brown PO, Botstein D. Molecular portraits of human breast tumors. Nature 2000; 406:747-752.

10. Cadoo KA, Fornier NM, Morris PG. Biological subtypes of breast cancer: currents concepts and implications of recurrence patterns. QJ Med Mol Imaǵing 2013; 57:312-321.

11. Arrechea MA, García FV, Córdoba AI, Ibáñez BB, Santamaría MM, Guillén FG. Subtipos moleculares del cáncer de mama: implicaciones pronósticas y características clínicas e inmunohistoquímicas. An Sist Sanit Navar 2011; 34:219-233.

12. Walerych D, Napoli M, Collavin L, Del Sal G. The rebel angel: mutant p53 as the driving oncoǵene in breast cáncer. Carcinogenesis 2012; 33:2007-2017.

13. Friedrichs K, Gluba S, Eidtrnann H, Jonat W. Overexpression of p53 and proǵnosis in breast cancer. Cancer 1993; 72:3641-3647.

14. Bottini A, Berruti A, Bersiga A, Brizzi MP, Brunelli A, Gorzeǵno G, DiMareo B, Aguǵgini S, Bolsi G, Cirillo F, Filippini L, Betri E, Bertoli G, Alquati P, Dogliotti L. p53 but not bel-2 immunostaining is predictive of poor clinical complete response to primary chemotherapy in breast cancer patients. Clin Cancer Res 2000; 6:2751-2758.

15. Eom YH, Kim HS, Lee A, Song BJ, Chae BJ. Bel2 as a subtype-specific prognostic marker for breast cancer. J Breast Cancer 2016; 19:252-260.

16. El-Hafez AA, El-aty SM, Basem HE. Different prognnostic factors correlate with bel2 expression among triple negative and nontriple neǵative breast cancers. Asian Pacific J Cancer Prev 2013 ;14:1037-1041.

17. Callagy GM, Webber MJ, Pharoah PD, Caldas C. Meta-analysis confirms bel2 is an independent prognostic marker in breast cancer. BMC Cancer 2008; 8:153-163.

18. Zubair M, Hashmi S, Afzal S, Muhammad I, Ud Din H, Ahmed R. Immunohistochemical expression of $\mathrm{B}$ cell lymphoma- 2 with clinicopathological correlation in triple negative breast cancers in northern Pakistan. Asian Pac J Cancer Prev 2016; 17:3619-3622.
19. Elston CW, Ellis IO. Pathological proginostic factors in breast cancer. I. The value of histological grade in breast cancer; experience from a large study with long-term follow-up. Histopathology 1991; 19:403-410.

20. Hammond ME, Hayes DF, Dowsett M, Allred DC, Hagerty KL, Badve S, Fitzgibbons PL, Francis G, Goldstein NS, Hayes M, Hicks DG, Lester S, Love R, Mangu PB, MeShane L, Miller K, Osborne CK, Paik S, Perlmutter J, Rhodes A, Sasano H, Schwartz JN, Sweep FC, Taube S, Torlakovic EE, Valenstein $P$, Viale $G$, Visscher $D$, Wheeler T, Williams RB, Wittliff JL, Wolff AC. American Society of Clinical Oncology/ College of American Pathologists guideline recommendations for immunohistochemical testing of estrogen and progesterone receptors in breast cancer. J Clin Oncol 2010; 28:2784-2795.

21. Wolff AC, Hammond ME, Hicks DG, Dowsett M, McShane LM, Allison KH, Allred DC, Bartlett JM, Bilous M, Fitzǵibbons P, Hanna W, Jenkins RB, Mangu PB, Paik S, Perez EA, Press MF, Spears PA, Vance GH, Viale G, Hayes DF; American Society of Clinical Oncology; College of American Pathologists. Recommendations for human epidermal growth factor receptor 2 testing in breast cancer: American Society of Clinical Oncology / College of American Pathologists clinical practice guideline update. J Clin Oncol 2013; 31:3997-4013.

22. Reigosa A, Hardisson D, Sanz-Rodriguez F, Caleiras E, Saldivia F, Fernández A. Subclassification of the molecular types of breast cancer based on the expression of immunohistochemical markers and evolution. Invest Clin 2016; 57:187-216.

23. Zaha DC, Lazăr E, Lăzureanu C. Clinicopathologic features and five years survival analysis in molecular subtypes of breast cancer. Rom J Morphol Embryol 2010; 51:85-89.

24. Seong MK, Lee JY, Byeon J, Sohn YJ, Seol H, Lee JK, Kim EK, Kim HA, Noh WC. Bcl-2 is highly significant prognostic marker of hormone-receptor-positive, human epidermal growth factor receptor-2-negative breast cancer. Breast Cancer Res Treat 2015; 150:141-148.

Vol. 59(4): 325 - 338, 2018 
25. Coates AS, Winer EP, Goldhirseh A, Gelber RD, Gnant M, Piccart-Gebhart M, Thürlimann B, Senn HJ; Panel Members. Tailoring therapies-improving the management of early breast cancer: St Gallen International Expert Consensus on the primary therapy of early breast eancer 2015. Ann Oncol 2015; 26:1533-1546.

26. Honma N, Horii R, Ito Y, Saji S, Younes M, Iwase T, Akiyama F. Differences in clinical importance of Bel-2 in breast eancer according to hormone receptors status or adjuvant endocrine therapy. BMC Cancer 2015; 15:698-709.

27. Hwang KT, Han W, Kim J, Moon HG, Oh S, Song YS, Kim YA, Chang MS5, Noh DY. Prognostic influence of bel2 on molecular subtypes of breast cancer. J Breast Cancer 2017; 20:54-64.

28. Dawson SJ, Makretsov N, Blows FM, Driver KE, Provenzano E, Le Quesne J, Baglietto L, Severi G, Giles GG, McLean CA, Callagy G, Green AR, Ellis I, Gelmon K, Turashvili G, Leung S, Aparicio S, Huntsman D, Caldas C, Pharoah P. BCL2 in breast cancer: a favorable prognostic marker across molecular subtypes and independent of adjuvant therapy received. Br J Cancer 2010; 103:668-675.
29. Abdel-Fatah TM, Dickinson PD, Moseley P, Reis-Filho JS, Green AR, Ellis IO. Bel2 as a surrogate prognostic and predictive marker in triple-negative breast cancer. J Clin Oncol 2011; 29:1024-1034.

30. Larsen MS1, Bjerre K, Giobbie-Hurder A, Lænkholm AV, Henriksen KL, Ejlertsen B, Lykkesfeldt AE, Rasmussen BB. Prognostic value of $\mathrm{Bcl}-2$ in two independent populations of estrogen receptor positive breast eancer patients treated with adjuvant endocrine therapy. Acta Oncol 2012; 51:781-789.

31. Abd El-Hafez A, Shawky Mohamed Ael-A, Elesawy BH. Different prognostic factors correlate with Bcl-2 expression among triple negative and non-triple negative breast cancers. Asian Pac J Cancer Prev 2013; 14:1037-1041. 